\title{
Optimum design for the ballistic diode based on graphene field-effect transistors
}

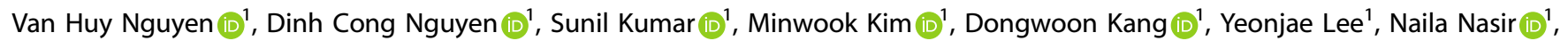
Malik Abdul Rehman ${ }^{1}$, Thi Phuong Anh Bach ${ }^{1}$, Jongwan Jung ${ }^{1}$ and Yongho Seo $\mathbb{D}^{1 凶}$

We investigate the transport behavior of two-terminal graphene ballistic devices with bias voltages up to a few volts suitable for electronics applications. Four graphene devices based ballistic designs, specially fabricated from mechanically exfoliated graphene encapsulated by hexagonal boron nitride, exhibit strong nonlinear $I-V$ characteristic curves at room temperature. A maximum asymmetry ratio of 1.58 is achieved at a current of $60 \mu \mathrm{A}$ at room temperature through the ballistic behavior is limited by the thermal effect at higher bias. An analytical model using a specular reflection mechanism of particles is demonstrated to simulate the specular reflection of carriers from graphene edges in the ballistic regime. The overall trend of the asymmetry ratio depending on the geometry fits reasonably with the analytical model.

npj 2D Materials and Applications (2021)5:89; https://doi.org/10.1038/s41699-021-00269-2

\section{INTRODUCTION}

Graphene, a two-dimensional (2D) material, is a useful material for electronic devices due to its excellent transport, mechanical, thermal, and optoelectronic properties ${ }^{1-4}$. The performance of devices, based on graphene field-effect transistors on $\mathrm{Si} / \mathrm{SiO}_{2}$ was limited by scattering from impurities, doping effect, charged surface states, surface roughness, and surface phonons ${ }^{5-8}$. Recently, hexagonal boron nitride (BN) has been used as an ideal substrate for graphene devices because of its flat surface and crystal structure similar to graphene ${ }^{9-11}$. With the development of fabrication techniques for BN encapsulation and one-dimensional contacts, the carrier mobility of graphene was measured up to $\sim 200,000 \mathrm{~cm}^{2} \mathrm{~V}^{-1} \mathrm{~s}^{-1}$ at room temperature ${ }^{12,13}$. This remarkable operation performance permits graphene to have a long carrier mean-free path $(\lambda)$, which is required for a graphene-based ballistic transport device (GBTD).

GBTDs based on geometric asymmetry have recently attracted considerable attention. Unlike conventional diodes and transistors, GBTDs do not need a sizable band gap to operate, but its critical dimensions should be on the order of or less than $\lambda^{14}$ and no scatterings are required except at graphene edges in order to achieve the ballistic behavior ${ }^{15,16}$. With promising features like high rectification efficiency, high responsivity, and low parasitic capacitance, GBTDs can be operated at $\mathrm{GHz} / \mathrm{THz}$ frequencies ${ }^{17-19}$. These devices can be adopted for terahertz detection and imaging, optical rectennas, optical heterodyne detection, current rectifiers, and biomedical diagnostics ${ }^{20-22}$. Several GBTDs were fabricated and demonstrated the ballistic transport properties, including geometric diodes ${ }^{23-27}$, graphene ballistic rectifiers ${ }^{17-}$ 19,28 , and geometric diodes for energy harvesting ${ }^{29,30}$. All reports show that the geometric features of graphene devices affect electron transport in the ballistic regime. However, the rectification efficiency and responsivity $(0.22-0.24 \mathrm{~A} / \mathrm{W})$ are low ${ }^{24-26}$ due to unwanted doping and impurities on the graphene during fabrication processes. Many theories have been studied to predict the increased responsivity with a wide difference in carrier mobilities using extended Landauer-Büttiker formalism. The geometric dependence of graphene ballistic rectifiers has been studied in the nonlinear transport regime ${ }^{18}$. The formalism of nonlinear transport in mesoscopic conductors based on extended multiterminal Landauer-Büttiker formulas ${ }^{31,32}$ was presented to treat two-terminal devices at a finite applied voltage and temperature ${ }^{33}$. From Dirac-like equations, the geometrically induced rectification of graphene was found to be ambipolar ${ }^{29}$. The properties of the nanodevice are supposed to be very sensitive to the geometric shape of the nanostructure. So far, geometric designs for optimizing rectification of GBTDs are indistinct and still under debate. The geometry diodes need no potential barrier, implying that it operates with zero-threshold voltage for the energy harvesting application.

In this paper, we report the fabrication and electrical characterization of two-terminal GBTD fabricated from mechanically exfoliated graphene with different geometries operating at room temperature. These GBTDs can be considered as a geometric diode because of their asymmetric behavior depending on the direction of the current. Using a modified fabrication in nanoscale, the achieved results of GBTDs exhibit nonlinear electrical transports with high rectification efficiency. The DC current-voltage and a gate electrode were used to tune the I-V characteristics and charge carriers with the applied back-gate voltage $\left(V_{g}\right)$. The ballistic transport characteristics of GBTDs are compared with numerical simulation based on the ballistic motion of particles. This study offers a pathway to optimize the other GBTDs and holds promise for potential application as graphene rectifiers and energy harvesting devices.

\section{RESULTS AND DISCUSSIONS Design and concept}

GBTDs behave like a diode due to their asymmetric structure. Figure 1 (a) shows a series of four designs of GBTDs, devices $A, B, C$, and $D$, with different geometries investigated in this study. All devices were designed to have the same tapered angle of around $30^{\circ}$ in the forward direction and different angles in the backward. From the simulation result (Supplementary Fig. 8(d)), the device performance around this angle was estimated to be high enough,

\footnotetext{
${ }^{1}$ Department of Nanotechnology and Advanced Materials Engineering, and HMC, Sejong University, 05006 Seoul, South Korea. ${ }^{凶}$ email: yseo@sejong.ac.kr
} 
(a)
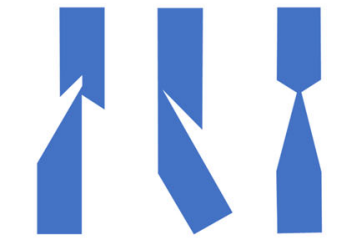

Device A

Device B Device C Device D

(b)

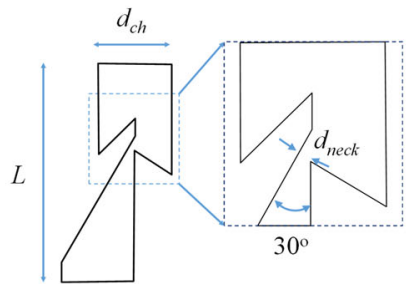

(c)

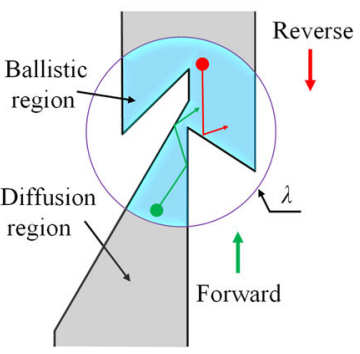

Fig. 1 Geometric designs and operation of ballistic transport devices. a Designs for ballistic devices with different geometries. b Configuration of device A shows dimensional parameters: length $(L)$, channel width $\left(d_{c h}\right)$, neck width $\left(d_{\text {neck }}\right)$, and tapered angle $\left(30^{\circ}\right)$. c The forward and backward directional motions of charge carriers in the ballistic regime. The ballistic region (blue region) is assumed to be inside a circle of radius $\lambda$, and the diffusive region (grey region) is outside the circle.

and the dimension of the device was suitable. These GBTDs were operated under an electric field given by the gate voltage $\left(V_{g}\right)$, altering the carrier density in the channel. These devices were made from the monolayer graphene encapsulated by a few-layer hexagonal boron nitride (BN) on a silicon oxide/silicon substrate. The basic physical requirement for GBTD is that its minimum feature size must be of the order of or smaller than the mean free path $(\lambda)$ of the charge carriers in graphene $23,34,35$, and graphene has a long $\lambda(\sim 1 \mu \mathrm{m})$ at room temperature ${ }^{1,19,36}$.

Figure $1(\mathrm{~b})$ shows the design of device $A$, where the critical dimension is the width of the neck $\left(d_{\text {neck }}\right)$, and the other parameters are length $(L)$, channel width $\left(d_{\mathrm{ch}}\right)$, and tapered angle. In this model, the charge carrier moving toward the neck is considered ballistic, and the geometry and boundaries of the device have an impact on the transmission probability. The geometry of GBTDs is designed to allow charge carriers onedirectional preference, acting as a funnel with a smooth flow and a restricted flow in opposite directions. The charges undergoing ballistic transport suffer collision and specular reflection at the edges of graphene as shown in Fig. 1(c). The charge carriers can move easily from the slowly tapered region (forward direction marked in green), while the carriers moving from the opposite direction may have difficulty in passing the abrupt constriction (backward direction marked in red). On the other hand, the effect of GBTDs depends on the majority carrier type (electrons or holes) of graphene controlled by $V_{g}$, as well as the geometric structure.

\section{Performance ballistic diodes}

Four GBTDs with graphene encapsulated by BN were fabricated on a Si chip as shown in Fig. 2(d). A detailed FESEM image of device A having $d_{\text {neck }} \approx 200 \mathrm{~nm}$ exhibits the constricted region of the device in submicron scale as indicated in Fig. 2(e). The sharp edges of the BNG-BN layer are clearly seen and are adequate for the device operating in the ballistic regime. Figure $2(\mathrm{f})$ and $(\mathrm{g})$ show representative examples of transfer curves with resistance versus $V_{g}$ to compare the devices prepared by modified and conventional methods (without edge defect treatment), respectively. The modified device exhibited minor hysteresis after $24 \mathrm{~h}$ in a vacuum $\left(1 \times 10^{-5}\right.$ Torr). The Dirac point (DP) of the device is at around $V_{g}=-1 \mathrm{~V}$, which was slightly shifted toward $n$-type doping. On the other hand, the conventionally fabricated sample (Fig. 2(g)) showed a large hysteresis shifting DP into a heavily $n$-doped state due to impurity ${ }^{37,38}$. The carrier mobilities have been determined from the slope $\frac{d V_{s d}}{d V_{g}}$ of the transfer characteristics curve of the graphene-based field-effect transistor at room temperature (Supplementary Fig. 4), giving values of $130,000 \mathrm{~cm}^{2} \mathrm{~V}^{-1} \mathrm{~s}^{-1}$ and $47,000 \mathrm{~cm}^{2} \mathrm{~V}^{-1} \mathrm{~s}^{-1}$ for electrons and holes, respectively. $\lambda$ is estimated from the equation, $\lambda=\hbar / 2 e \mu \sqrt{n_{\text {th }} / \pi^{12,18}}$, which gives $\lambda \approx 0.7 \mu \mathrm{m}$, where $\hbar$ is Planck's constant, $\mathrm{e}$ is the electron charge, $n_{t h}$ is the threshold carriers density depends on $V_{g},\left|n_{t h}\right|=\left|a \times\left(V_{g, t h}-V_{g, 0}\right)\right|$ and $a=7.2 \times 10^{10} \mathrm{~cm}^{-2}$
$\mathrm{V}^{-1}$ is the back gate lever $\mathrm{arm}^{39}$. These figures of merit of graphene quality ensure that the GBTDs can be operated in a ballistic transport regime. Of the 50 samples fabricated using conventional methods, only 5 samples showed ballistic behavior, whereas 12 samples were valid among 13 samples fabricated using the modified method.

Figure 3(a) and (b) show the I-V characteristic curves of device A with majority carrier at $V_{g}=+2.5 \mathrm{~V}$ and $-5 \mathrm{~V}$, respectively. At $V_{g}=$ $+2.5 \mathrm{~V}$ with the $n$-type carrier, the $I-V$ curve of the device is nonlinear, exhibiting that $V_{\mathrm{sd}}=+1.3 \mathrm{~V}$ was measured at $I_{\mathrm{sd}}=+100 \mu \mathrm{A}$, while $V_{s d}=-1.1 \mathrm{~V}$ was measured at $I_{\mathrm{sd}}=-100 \mu \mathrm{A}$. At $V_{g}=-5 \mathrm{~V}$ with p-type carriers, the nonlinear behavior is shown in the opposite direction where the higher absolute value of $V_{\text {sd }}$ was measured at $I_{\text {sd }}=-100 \mu \mathrm{A}$. This asymmetric characteristic is evidence that the device was operated in the ballistic regime. However, the I-V curve was slightly bent when higher currents were applied for both negative and positive current biases, which can be explained by the heating effect in a nanodevice at room temperature ${ }^{40,41}$.

To investigate the rectification ratio of the devices, $V_{g}$ was tuned at the fixed $l_{\mathrm{sd}}$ while carrier density was varied. As the direction of carrier motion in the devices depends on the charge carrier type, $V_{g}$ was employed to change the majority charge carrier type. We define an asymmetric ratio $(\gamma)$ as the ratio of the measured voltages $\left(V_{\mathrm{sd}}\right)$ between positive $\left(+I_{\mathrm{sd}}\right)$ and negative currents $\left(-I_{\mathrm{sd}}\right)$, which can be expressed as:

$\gamma=\left|\frac{V\left(+I_{s d}\right)}{V\left(-I_{s d}\right)}\right|$.

If $\gamma>1$, the electron transport is dominant, and the hole transport is dominant in case of $\gamma<1$ in Eq. (1). The $\gamma$ was plotted as a function of $V_{g}$ as shown in Fig. 3(c). Here, $V_{g}$ at the DP $\left(V_{D}\right)$ is around $-2 \mathrm{~V}$, having zero conductivity as there is no carrier. $\gamma$ is $>$ 1 for $V_{g}>V_{\mathrm{D}}$ and $<1$ for $V_{g}<V_{\mathrm{D}}$ with the maximum value of $\gamma=$ 1.22 at $V_{g}=+2.5 \mathrm{~V}$. $\gamma$ has peaks around the $V_{g}=+2.5 \mathrm{~V}$ and $-5 \mathrm{~V}$ with charge carriers of electrons and holes, respectively, and vanishes as it moves away from DP. Notably, the electron transport has a higher $\gamma$ than the hole transport due to higher carrier mobility with longer $\lambda$. On the other hand, resistance change was found to depend on the channel voltage (Supplementary Fig. 5), which is further evidence of the ballistic characteristic of the device as the incident angles of the carriers are varied by the strength of the electric force. To evaluate the change of $\gamma$ with increasing current bias, the $\gamma$ versus the sourcedrain current $\left(I_{s d}\right)$ with different $V_{g}$ was plotted as shown in Fig. 3 (d). The $I_{\text {sd }}$ was applied within the $\pm 100 \mu \mathrm{A}$ range for the device to be stably operated avoiding possible damage. At the Dirac point of $V_{g}=-2 \mathrm{~V}$, the $\gamma \approx 1$ while the $\gamma$ was deviated from 1 and almost monotonically increased with increasing current at $V_{g}=+2.5 \mathrm{~V}$ and $-5 \mathrm{~V}$. This increment is not related to the self-heating effect because the heating effect cannot depend on the current direction. This can be explained by the fact that increasing current raises the directional uniformity of ballistic charges in graphene with a strengthened electric field. Furthermore, it can be 
(a)

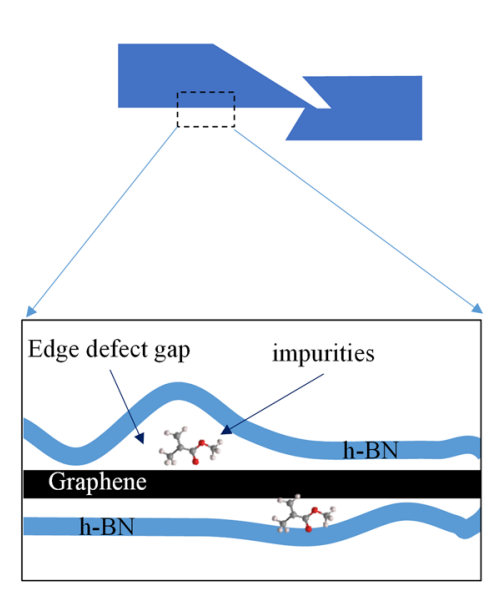

(b)

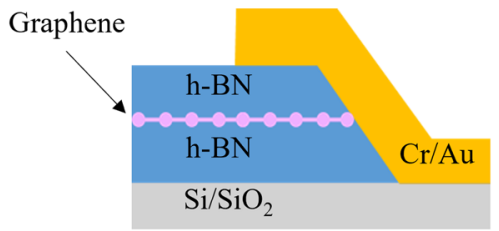

(d)

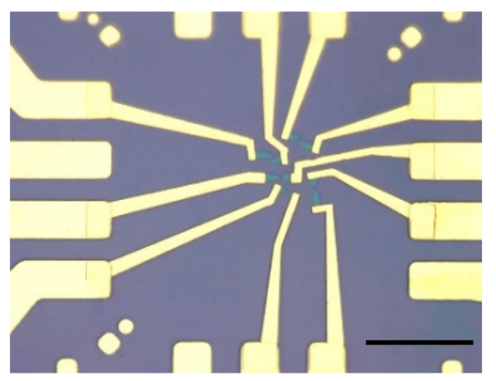

(c)

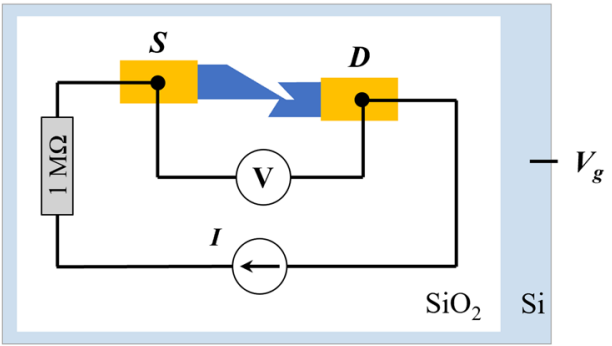

(e)

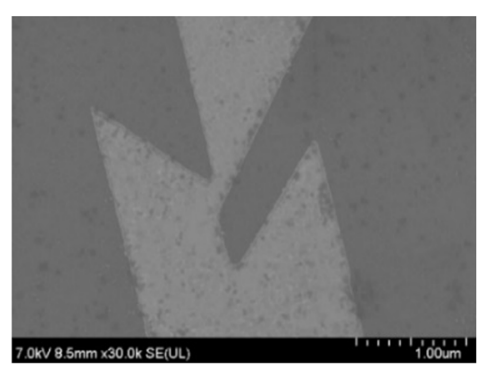

(f)

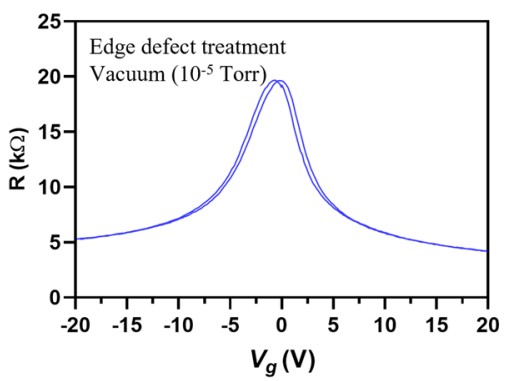

(g)

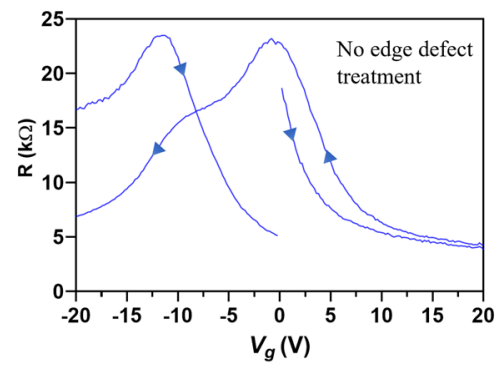

Fig. 2 Fabrication process and characteristics of the sample. a Ballistic device with BN-Gr-BN layer was fabricated by RIE, the cross-section of which may include contaminations. b One-dimensional edge contact structure of the graphene encapsulated with BNs. $\mathbf{c}$ DC current-voltage measurement configuration is illustrated. The current was supplied with a series resistance of $1 \mathrm{M} \Omega$ to provide source-drain current $\left(I_{s d}\right)$. $V_{g}$ was applied directly to the silicon substrate to control the carrier type and carrier concentration. $\mathbf{d} 100 \times$ optical microscopic image of a GBTD. Scale bar $=30 \mu \mathrm{m}$. e FESEM image of the device A shows a narrow neck width. Scale bar $=1 \mu \mathrm{m}$. $\mathbf{f}-\mathbf{g}$ Transfer curves of resistance versus $V_{g}$ of the GBTDs fabricated by the modified (f) and conventional method $(\mathbf{g})$, respectively.

interpreted that applying a high field renders reflections at the edges specular ${ }^{19}$. The localized edge states exist near disordered edges with dangling bond terminations, causing the Coulomb charging effect in the graphene channel ${ }^{42-46}$. When a low current is applied, the disordered edge causes diffuse reflection for ballistic charges. At a high current, the high electric field provides sufficient energy to the charge carriers to pass into the localized area. Initial carriers are trapped at the edges, and subsequent carriers will be reflected by the trapped charges in a specular way.

To investigate the geometric effect on the transport properties more evidently, device $B$ with a modified design was prepared, and its $I-V$ curves at $V_{g}=+17 \mathrm{~V}$ and $-2 \mathrm{~V}$ are shown in Fig. 4(a). It has a long straight edge on one side, and the opposite side has an obtuse angle at the neck for the backward direction. With this special design at the neck region, the device exhibited non-linear $I-V$ characteristic curves with a high $\gamma$. A common feature between the $I-V$ curves for electron and hole carriers is noticeable, as it is linear in negative $V_{\text {sd }}$ and strongly bent in positive $V_{\text {sd }}$. The increment of charge carriers renders specular reflection at the boundary due to the long-range Coulomb effect as mentioned in device A. The $\gamma$ of the device was plotted as a function of $V_{g}$ as shown in Fig. 4(b). It was found that near the Dirac point, $y$ has value $\approx 1$, which increases with $\pm\left|V_{g}-V_{D}\right|$. The highest value of $\gamma$ $=1.58$ was found at $I_{s d}=60 \mu \mathrm{A}$, and $V_{g}$ at peak $\gamma$ was strongly shifted by $I_{\text {sd }}$. This further clarifies the intensified specular reflection at the edges by high currents. The reason why $\gamma$ of device $B$ is higher than that of device $A$ can be found from the wide structure in the backward input channel. As device $B$ has a wider angle and width in backward input, the reflected carriers from the edges were spread, lowering the probability to pass the neck. It should be considered that the edge is not perfectly flat, causing partially diffusive reflections. Furthermore, the mean free path is not long enough to move far away from the neck once it is reflected.

To investigate the geometric dependence of the efficiency, we investigate other devices. Figure 5(a) displays the $I-V$ characteristic curves of device $C$ as a function of bias current with the fixed $V_{g}=$ $+2 \mathrm{~V}$ and $-2 \mathrm{~V}$. The $I-V$ curves of device $C$ are also non-linear depending on $V_{g}$, similarly to the devices $\mathrm{A}$ and $\mathrm{B}$. As the current is increased, $\gamma$ increases up to 1.32 as shown in Fig. 5(b).

Figure 5(c) shows the $I-V$ characteristic curves of device $D$ with majority charge carriers of electron and hole at $V_{g}=+18 \mathrm{~V}$ and $-2 \mathrm{~V}$. Here, the higher $\gamma$ than device $C$ is achieved from the nonlinear $I-V$ characteristic curves. This is plausible considering that the backward resistance is increased due to the sharp angle at the neck in the right-hand region in the asymmetric structure. It is found that the forward direction of the device was markedly reversed by $V_{g}$. The device exhibits quite linear curves within $I_{\text {sd }}=$ $\pm 20 \mu \mathrm{A}$ range and became non-linear with $I_{\mathrm{sd}}> \pm 20 \mu \mathrm{A}$ for both electron and hole carriers. The current-dependent effect was analyzed from $\gamma$ versus $I_{\text {sd }}$ curves measured at $V_{g}=+18 \mathrm{~V}$ and -2 $V$ as shown in Fig. 5(d). It is noted that the behavior of current bias has four distinct states (I, II, III, IV) for both electrons and holes. The $\gamma$ is slowly increased for $I_{\text {sd }}<20 \mu \mathrm{A}(\mathrm{I})$, rapidly increased for $20<I_{\text {sd }}$ $<70 \mu \mathrm{A}$ (II), saturated for $70<I_{\text {sd }}<90 \mu \mathrm{A}$ (III), and slowly decreased for $90<I_{\text {sd }}<100 \mu \mathrm{A}$ (IV). The highest $\gamma=1.66$ is obtained at $I_{\text {sd }}=$ 
(a)
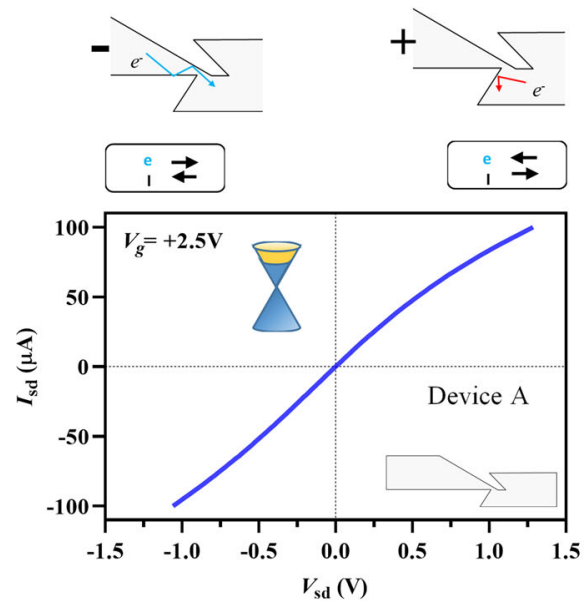

(c)

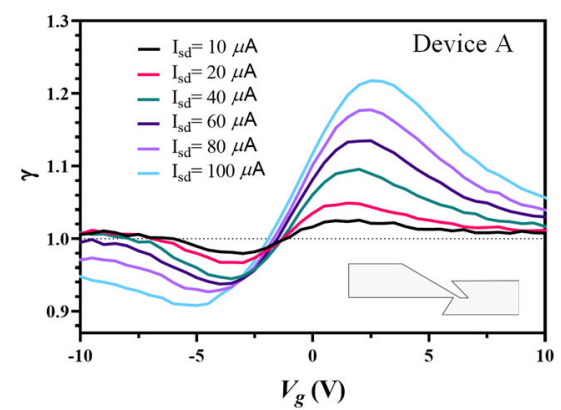

(d)
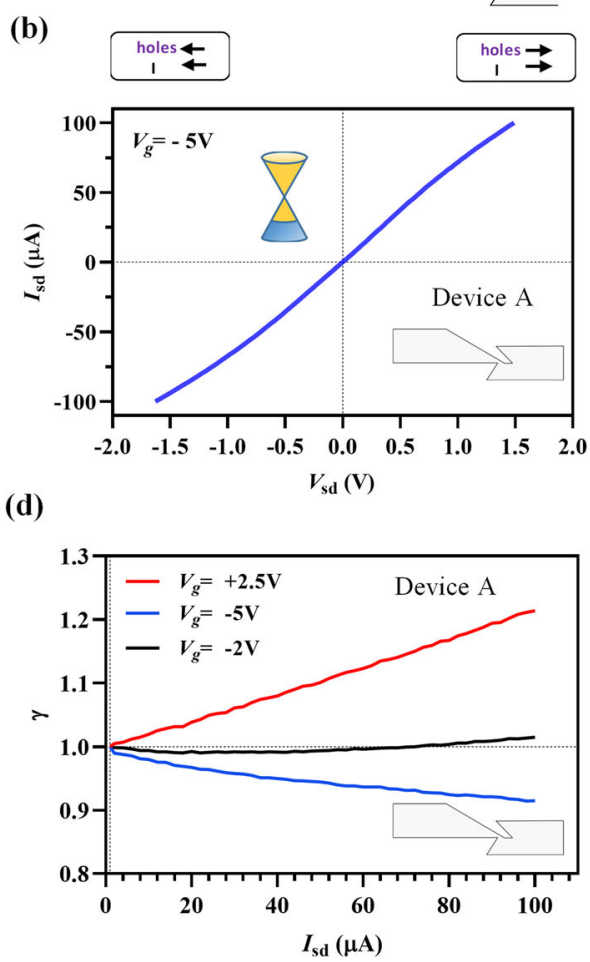

Fig. 3 Transport properties of device $\mathbf{A}$ at room temperature. $\mathbf{a}, \mathbf{b} l-V$ characteristics at $V_{g}=+2.5 \mathrm{~V}$ and $-5 \mathrm{~V}$, respectively. c The $\gamma$ was plotted as a function of $V_{g}$ at different $I_{\text {sd }}$. d The $\gamma$ versus $I_{\text {sd }}$ at $V_{g}=+2.5 \mathrm{~V},-5 \mathrm{~V}$, and $-2 \mathrm{~V}$.

(a)

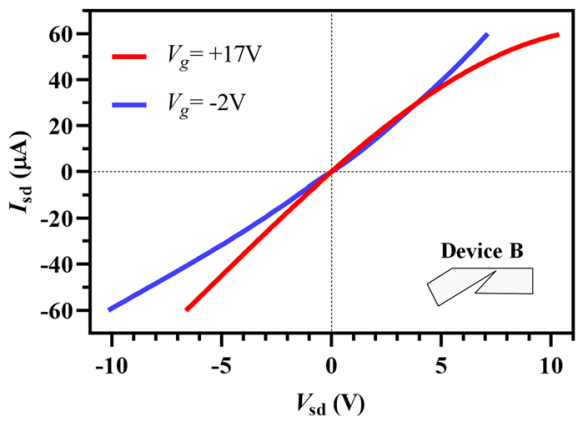

(b)

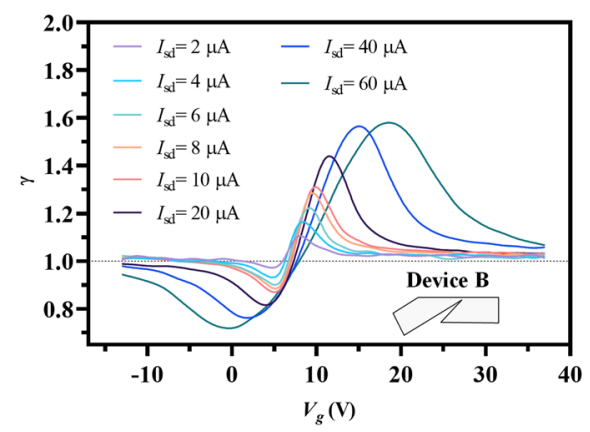

Fig. 4 Transport properties of device $\mathbf{B}$ at room temperature. a $l-V$ curves of device $\mathrm{B}$ at $V_{g}=+17 \mathrm{~V}$ and $-2 \mathrm{~V}$. $\mathbf{b}$ The $\gamma$ was measured as a function of the $V_{g}$ at various currents $I_{\text {sd. }}$.

$80 \mu \mathrm{A}$, which is increased further by increasing the current bias, implying that $\gamma$ is limited in the ballistic regime. As the power dissipation given by $P=I . V$ in region IV is higher than those for other devices, a slight decrement of $\gamma$ is attributed to the selfheating effect. In a high bias regime, the carriers are no longer ballistic, and hot electrons are induced and scattered by surface phonons in graphene ${ }^{47-49}$.

The highest values of $\gamma$ along with geometry parameters for different types of ballistic devices are listed in Table 1. Due to the inaccuracy of the lithographic process, the dimension of each device varies slightly. Device B has higher $\gamma$ than device A, and device $D$ has higher $\gamma$ than device $C$, though they have similar shapes. A common feature of $B$ and $D$ is a wider area in backward insertion, rendering the reflected charges spread easily. As device $D$ has a typical shape for the ballistic rectifier, its efficiency can be compared with the previously published result. The highest value of $\gamma=1.66$ of device $D$ is much higher than that of the earlier report $(\gamma=1.31)^{24}$ and $(\gamma=1.4)^{26}$.

\section{Numerical simulation}

Numerical simulation was performed, modifying the model suggested by Zhang and Hung to compare ballistic efficiencies of geometric devices qualitatively ${ }^{50,51}$. In Fig. 6(a), the ballistic region was considered as a semicircle with a radius $\lambda$, the center of which is the neck of the device. The charges in the green (yellow) region contribute to the forward (backward) transmission. The grey region is a dead region that may not contribute to the backward transmission. We assume that the charges can pass the neck either directly or after repeated specular reflections at the edges of the device as shown in Fig. 6(b). The allowed angles 
(a)

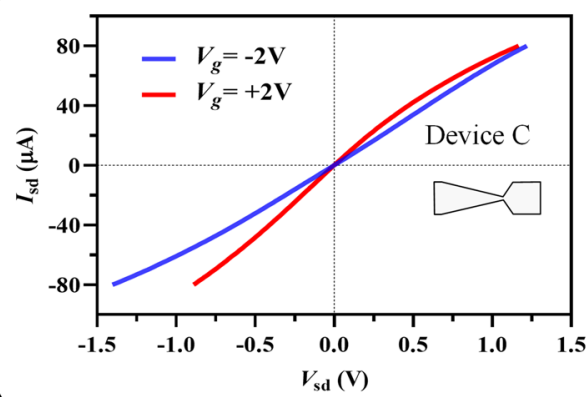

(c)

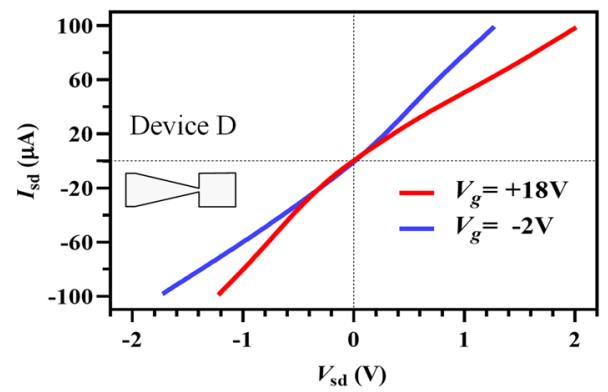

(b)

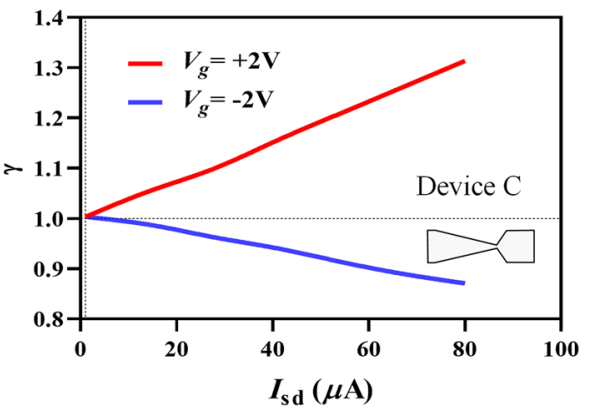

(d)

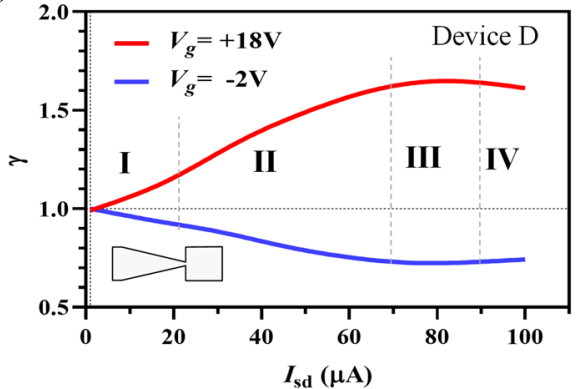

Fig. 5 Transport properties of devices $\mathbf{C}$ and $\mathbf{D}$ at room temperature. $\mathbf{a} I-V$ curves of device $C$ are plotted as a function of $I_{s d}$ at $V_{g}=+2 \mathrm{~V}$ and $-2 \mathrm{~V}$. $\mathbf{b} \gamma$ of device $C$ is shown as a function of $I_{\mathrm{sd}}$. $\mathbf{c}$ The $I-V$ characteristic curves of device $\mathrm{D}$ at $V_{g}=-2 \mathrm{~V}$ and $+18 \mathrm{~V}$. $\mathbf{d} \gamma$ of device $\mathrm{D}$ is shown as a function of $I_{\text {sd }}$.

Table 1. The highest $\gamma$ are listed with geometric parameters of different ballistic devices.

\begin{tabular}{lllllll}
\hline & $L(\mu \mathrm{m})$ & $d_{c h}(\mu \mathrm{m})$ & $d_{\text {neck }}(\mathrm{nm})$ & Current bias $(\mu \mathrm{A})$ & Highest $\gamma$ \\
\hline Device A & 10 & 2 & $190 \pm 5$ & 100 & 1.22 & 1.58 \\
Device B & 8 & 2 & $125 \pm 5$ & 60 & 1.32 \\
Device C & 6 & 2 & $190 \pm 5$ & 80 & 1.66 \\
Device D & 6 & 2 & $175 \pm 5$ & 80 & $1.31^{24} ; 1.4^{26}$ \\
\hline
\end{tabular}

can be easily calculated using a mirroring process. The initial ballistic region $\left(M_{0}\right)$ is expanded into $M_{1}$ and $M_{2}$ by mirroring at the edge line to include the specular reflection cases. The allowed angle intervals, $\theta_{1}^{\min } \leq \theta \leq \theta_{1}^{\max }$ and $\theta_{2}^{\min } \leq \theta \leq \theta_{2}^{\max }$, from $M_{0}$ can be obtained by considering straight lines (dotted) from the mirror points in $M_{1}$ and $M_{2}$. All possible charge carriers in the $A B C D$ region are integrated, and a Gaussian distribution function with the standard deviation $(\sigma)$ and the mean $(\bar{a})$ is adopted to represent the ballistic angle distribution of the charges. The charges are assumed to be uniformly distributed in the area, and the integration intervals are depicted in Fig. 6(c-d).

The transmission ratio $(\delta)$ was defined as the ratio of transmission probabilities for forward and reverse biases (see Supplementary Figs. 6 and 7 for detail). Figure 7(a) displays $\delta$ for different values as a function of the $d_{\text {neck }}$ for the various devices depicted in Fig. 1 (a) with $\sigma=0.55 \mathrm{rad}, \bar{a}=0$ (for device $C$ and D), $\bar{a}$ $=7 \pi / 18 \mathrm{rad}$ (for device $\mathrm{A}$ and $\mathrm{B}$ ) and $\lambda=0.25 \mu \mathrm{m}$. The simulation results show that $\delta$ is increased monotonically with decreasing $d_{\text {neck. }}$. The model also indicates that $\delta$ depends strongly on $\lambda$, angular distribution, and geometry of the device. From LandauerBüttiker formalism, $\delta$ can be approximated to $\gamma$, as it represents the asymmetry between the forward and backward directions ${ }^{51}$

Figure 7 (b) shows the experimental results of $\gamma$ compared with the simulation results of $\delta$. The experimental results were measured at $40 \mu \mathrm{A}$ bias current as it was not affected by the heating effect. In principle, $\gamma$ is expected to be equal to $\delta$, and it can be seen that the overall trend of $\gamma$ depending on the geometry fits reasonably with the analytical model. The experimentally measured $\gamma$ is lower than the numerically estimated $\delta$. Usually, theory predicts a larger rectification ratio as compared to the experiment. For example, El-Araby et al. ${ }^{30}$ predicted the asymmetry of $\sim 10$, and Berdiyorov et al. predicted $\sim 5 .^{27}$ Particularly, Stearns and Moddel predicted $\sim 5$ with geometry similar to device $A,{ }^{52}$ where the drift velocity was parallel to the direction of the applied voltage. However, the average orientation of the velocity in our simulation was set as the direction from the center of the allowed region to the neck because it was the overall drifting direction of the current. On the other hand, the experimental values for the current rectification do not exceed 2. Furthermore, the reflection from the graphene edge may be diffused partially due to non-uniformly etched edge lines with contaminations as mentioned in the fabrication section. These diffusion phenomena may reduce the rectification efficiency strongly as shown in the angular distribution effect (Supplementary Figs. 10, 11).

In this report, we have modified the fabrication process for encapsulated graphene to improve the quality of graphene, and corresponding GBTDs with different geometries were fabricated using electron-beam lithography. The devices exhibited ballistic behavior at room temperature. It was found that the GBTD devices with different geometries exhibit asymmetric $I-V$ characteristics. It was shown that ballistic transport can be altered and enhanced by optimizing the geometries. The asymmetry ratio $\gamma$ was found up to $1.58\left(\right.$ at $\left.l_{\mathrm{sd}}=60 \mu \mathrm{A}\right)$ depending on the back-gate voltage and bias 
(a)
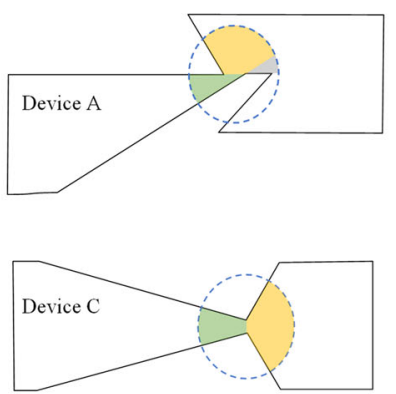

(c)

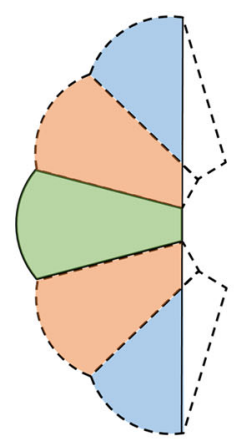

(b)
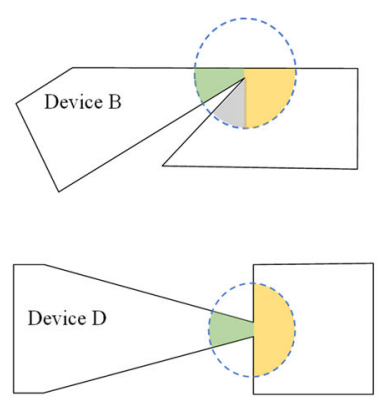

(d)

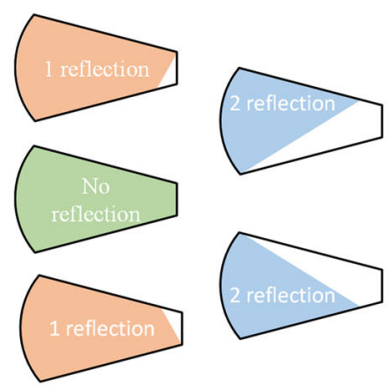

(b)

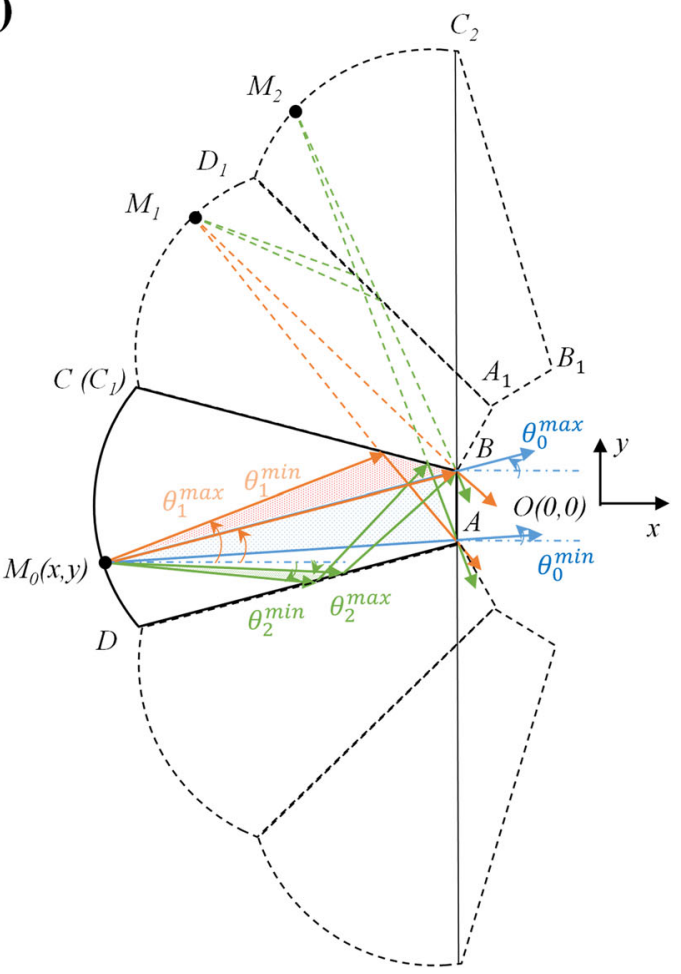

Fig. 6 Simulation model for transport of charge carriers in the ballistic region of devices. a Ballistic regions are denoted as dotted circles with the radius $\lambda$. b A charge at point $M_{0}$ can pass $A B$ through processes: (1) direct passing with angles from $\theta_{0}^{\min }$ to $\theta_{0}^{\max }$ (blue arrow), (2) reflecting once from edge line $(\overline{\mathrm{CB}})$ with the angle $\left(\theta_{1}^{\min } \leq \theta \leq \theta_{1}^{\max }\right.$ ) (orange arrow), or (3) reflecting twice from $\overline{\mathrm{AD}}$ and $\overline{\mathrm{CB}}$ with $\theta_{2}^{\min } \leq \theta \leq \theta_{2}^{\max }$ (green arrow). c The considered areas for passing the neck in forward transmission: direct transport (no reflection: green region); 1 reflection (orange region); 2 reflection (blue region). d Schematic representation of possible transmission areas to be integrated.

(a)

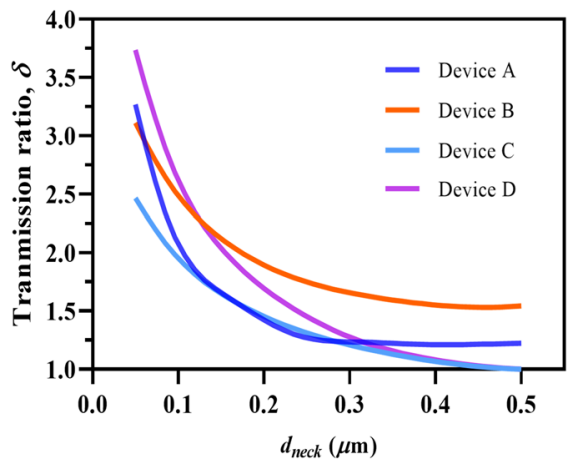

(b)

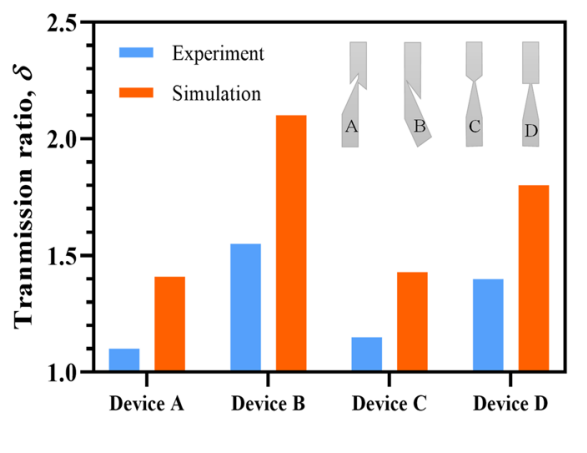

Fig. 7 Simulation and experimental results of graphene ballistic transport devices. a $\delta$ of devices (A, B, C, D) were calculated as a function of $\mathrm{d}_{\text {neck }}$ with $\sigma=0.55 \mathrm{rad}, \bar{a}=7 \pi / 18 \mathrm{rad}$ (for device $\mathrm{A}$ and $\mathrm{B}$ ), $\bar{a}=0 \mathrm{rad}$ (for device $\mathrm{C}$ and $\mathrm{D}$ ) and $\lambda=0.25 \mu \mathrm{m}$. b Comparing the $\delta$ of devices (A, B, C, D) with experimentally measured $\gamma$ at the bias current of $40 \mu \mathrm{A}$.

current. A numerical simulation for the ballistic devices was performed based on the specular reflection of the carrier from graphene edges in the ballistic regime. The overall trend of measured $\gamma$ from the $I-V$ characteristics agreed with the numerical simulation results. This study provides a pathway for optimizing the ballistic graphene rectifiers to be utilized as potential applications for energy harvesting devices.

\section{METHODS}

\section{Fabrication method}

GBTDs of different geometries were fabricated as shown in Fig. 1(a). The standard micromechanical exfoliation and dry-transfer method were used to prepare graphene ${ }^{53-55}$. To obtain graphene $(\mathrm{Gr})$ layer, Kish graphite (Covalent Materials Corp.) was mechanically exfoliated on $\mathrm{Si} / \mathrm{SiO}_{2}$ substrate with a $280 \mathrm{~nm}$ oxide layer using adhesive tape (Scotch Tape, $3 \mathrm{M}$ Inc). $\mathrm{Gr}$ and $\mathrm{BN}$ flakes were identified by using an optical microscope. A thin top $\mathrm{BN}$ flake of thickness $10-15 \mathrm{~nm}$ was similarly exfoliated on a cover glass with spin-coated polyvinyl alcohol (PVA)/polymethyl methacrylate (PMMA), which was used to pick up Gr and the bottom BN layer. The resultant BN/ $\mathrm{Gr} / \mathrm{BN}$ layer was transferred onto a p-type silicon substrate with $300 \mathrm{~nm}$ thermal oxide used as a global back-gate $\left(V_{g}\right)$ to tune the Fermi energy of $\mathrm{Gr}$ in the GBTD (Supplementary Fig. 1). The geometric designs were patterned by employing electron-beam lithography with a bilayer of PMMA $950 \mathrm{~K}(100 \mathrm{~nm}) / 495 \mathrm{~K}(100 \mathrm{~nm})$ and reactive ion etching (RIE) using a gas mixture of $\mathrm{SF}_{6}$ and $\mathrm{Ar}$. 
Some bubbles including residual gas or impurities appeared probably degrading graphene quality as illustrated in Fig. 2(a). The encapsulated graphene was etched by RIE as designed, and the samples were kept under a high vacuum $\left(1 \times 10^{-5}\right.$ Torr $)$ for $24 \mathrm{~h}$ to remove the trapped molecules below the bubbles near the edges of the device. AFM images before and after the vacuum treatment show a clear change of morphologies of the graphene channel (Supplementary Fig. 3). The source and drain electrodes were patterned with the PMMA $495 \mathrm{~K}(100 \mathrm{~nm}) / 950 \mathrm{~K}(100 \mathrm{~nm})$ resist. For the ohmic contacts, $\mathrm{Cr} / \mathrm{Au}$ $(5 \mathrm{~nm} / 60 \mathrm{~nm})$ were deposited by an electron-beam evaporator on the devices ${ }^{12}$, and a lift-off process was performed in warm acetone. Onedimensional edge-contacts were formed on the active graphene layer with low contact resistances as illustrated in Fig. 2(b). The device morphology was measured using field-emission scanning electron microscopy (FESEM, SU8010 Hitachi, Japan) and atomic force microscope (AFM, Nano focus Inc.). Raman spectroscopy was carried out using a micro-Raman system (Renishaw, InVia systems) with a laser beam of $514 \mathrm{~nm}$.

\section{Measurements}

The transport characteristics of two-terminal GBTDs were measured under a high vacuum $\left(1 \times 10^{-5}\right.$ Torr $)$ condition to remove the absorbed molecules under ambient air. Figure 2(c) shows the measurement configuration for the electrical characteristics. The back gate voltage $\left(V_{g}\right)$ was applied to control the carrier type and its concentration in the graphene channel by using a source-meter (Keithley, 2400-SMU). The bias current $\left(I_{s d}\right)$ was provided with a series resistor $(1 \mathrm{M} \Omega)$ between the source and drain terminals to avoid accidental electrical shock. A Keithley 2000 multimeter was used for measuring voltages $\left(V_{s d}\right)$ of devices. The graphene nanodevices suffered from hysteresis ${ }^{56,57}$ and self-heating effect at high bias $^{58,59}$ at room temperature and under ambient conditions. To avoid a thermal electromotive force and the offset problem in $I-V$ characteristic measurements, the source-drain currents were pulsed with a pulse width of $700 \mathrm{~ms}$ in the order of $0,+I_{1},-I_{1},+I_{2},-I_{2}, \ldots+l_{i},-I_{i}, 0$ by a computer control system.

\section{DATA AVAILABILITY}

The datasets generated during the current study are available from the corresponding author on reasonable request.

\section{CODE AVAILABILITY}

The codes generated during the simulation study are available from the corresponding author on reasonable request.

Received: 27 June 2021; Accepted: 29 October 2021; Published online: 02 December 2021

\section{REFERENCES}

1. Neto, A. C. et al. The electronic properties of graphene. Rev. Mod. Phys. 81, 109 (2009).

2. Lee, C., Wei, X., Kysar, J. W. \& Hone, J. Measurement of the elastic properties and intrinsic strength of monolayer graphene. Science 321, 385-388 (2008).

3. Ferralis, N. Probing mechanical properties of graphene with Raman spectroscopy. J. Mater. Sci. 45, 5135-5149 (2010).

4. Metzger, $C$. et al. Biaxial strain in graphene adhered to shallow depressions. Nano Lett. 10, 6-10 (2010).

5. Chen, J.-H. et al. Intrinsic and extrinsic performance limits of graphene devices on SiO 2. Nat. Nanotechnol. 3, 206-209 (2008).

6. Ando, T. Screening effect and impurity scattering in monolayer graphene. J. Phys. Soc. Jpn. 75, 074716-074716 (2006).

7. Ryu, S. et al. Atmospheric oxygen binding and hole doping in deformed graphene on a SiO2 substrate. Nano Lett. 10, 4944-4951 (2010).

8. Perebeinos, V., Rotkin, S. V., Petrov, A. G. \& Avouris, P. The effects of substrate phonon mode scattering on transport in carbon nanotubes. Nano Lett. 9 , 312-316 (2009)

9. Dean, C. R. et al. Boron nitride substrates for high-quality graphene electronics. Nat. Nanotechnol. 5, 722-726 (2010).

10. Gannett, W. et al. Boron nitride substrates for high mobility chemical vapor deposited graphene. Appl. Phys. Lett. 98, 242105 (2011).
11. Bediako, D. K. et al. Heterointerface effects in the electrointercalation of van der Waals heterostructures. Nature 558, 425-429 (2018).

12. Wang, L. et al. One-dimensional electrical contact to a two-dimensional material. Science 342, 614-617 (2013).

13. Bolotin, K. I. et al. Ultrahigh electron mobility in suspended graphene. Solid State Commun. 146, 351-355 (2008).

14. Beenakker, C. \& van Houten, H. Quantum transport in semiconductor nanostructures. Solid State Phys. 44, 1-228 (1991).

15. Mayorov, A. S. et al. Micrometer-scale ballistic transport in encapsulated graphene at room temperature. Nano Lett. 11, 2396-2399 (2011).

16. Masubuchi, S. et al. Boundary scattering in ballistic graphene. Phys. Rev. Lett. 109, 036601 (2012).

17. Auton, G. et al. Graphene ballistic nano-rectifier with very high responsivity. Nat. Commun. 7, 1-6 (2016).

18. Brownless, J., Zhang, J. W. \& Song, A. M. Graphene ballistic rectifiers: Theory and geometry dependence. Carbon 168, 201-208 (2020).

19. Singh, A. K., Auton, G., Hill, E. \& Song, A. Graphene based ballistic rectifiers. Carbon 84, 124-129 (2015)

20. Gadalla, M. N., Abdel-Rahman, M. \& Shamim, A. Design, optimization and fabrication of a $28.3 \mathrm{THz}$ nano-rectenna for infrared detection and rectification. Sci. Rep. 4, 1-9 (2014).

21. Zhu, Z., Joshi, S. \& Moddel, G. High performance room temperature rectenna IR detectors using graphene geometric diodes. IEEE J. Sel. Top. Quantum Electron. 20, 70-78 (2014).

22. Auton, G. et al. Terahertz detection and imaging using graphene ballistic rectifiers. Nano Lett. 17, 7015-7020 (2017).

23. Zhu, Z., Joshi, S., Grover, S. \& Moddel, G. Graphene geometric diodes for terahertz rectennas. J. Phys. D: Appl. Phys. Res. 46, 185101 (2013).

24. Moddel, G., Zhu, Z., Grover, S. \& Joshi, S. Ultrahigh speed graphene diode with reversible polarity. Solid State Commun. 152, 1842-1845 (2012).

25. Zhu, Z., Grover, S., Krueger, K. \& Moddel, G. in 2011 37th IEEE Photovoltaic Specialists Conference. 002120-002122 (IEEE)

26. Wang, H. et al. CVD-Grown Monolayer Graphene-Based Geometric Diode for $\mathrm{THz}$ Rectennas. Nanomaterials 11, 1986 (2021).

27. Berdiyorov, G. \& Hamoudi, H. Creating graphene geometry diodes through fluorination: First-principles studies. Comput. Mater. Sci. 188, 110209 (2021).

28. Auton, G., Kumar, R. K., Hill, E. \& Song, A. Graphene triangular ballistic rectifier: fabrication and characterisation. J. Electron. Mater. 46, 3942-3948 (2017).

29. Dragoman, D. \& Dragoman, M. Geometrically induced rectification in two dimensional ballistic nanodevices. J. Phys. D: Appl. Phys. Res. 46, 055306 (2013).

30. El-Araby, H. A., Malhat, H. A. E.-A. \& Zainud-Deen, S. H. Nanoantenna with geometric diode for energy harvesting. Wirel. Pers. Commun. 99, 941-952 (2018)

31. Buttiker, M. Capacitance, admittance, and rectification properties of small conductors. J. Condens. Matter Phys. 5, 9361 (1993).

32. Christen, T. \& Büttiker, M. Gauge-invariant nonlinear electric transport in mesoscopic conductors. Europhys. Lett. 35, 523 (1996).

33. Song, A. Formalism of nonlinear transport in mesoscopic conductors. Phys. Rev. $B$ 59, 9806 (1999).

34. Popov, V. \& Gorbunov, V. A possible method for studying fermi surfaces. Sov. Phys. J. 16, 517 (1963).

35. Jakobsen, H. A. Chemical reactor modeling 183-365 (Springer, 2014).

36. Novoselov, K. S. et al. Electric field effect in atomically thin carbon films. Science 306, 666-669 (2004).

37. Liu, H., Liu, Y. \& Zhu, D. Chemical doping of graphene. J. Mater. Chem. 21, 3335-3345 (2011).

38. Moser, J. et al. The environment of graphene probed by electrostatic force microscopy. Appl. Phys. Lett. 92, 123507 (2008).

39. Banszerus, L. et al. Ballistic Transport Exceeding $28 \mathrm{mu}$ m in CVD Grown Graphene. Nano Lett. 16, 1387-1391 (2016).

40. Liu, Y.-Y. et al. Effect of room temperature lattice vibration on the electron transport in graphene nanoribbons. Appl. Phys. Lett. 111, 133107 (2017).

41. Liao, A. D. et al. Thermally limited current carrying ability of graphene nanoribbons. Phys. Rev. Lett. 106, 256801 (2011).

42. Silvestrov, P. \& Efetov, K. Charge accumulation at the boundaries of a graphene strip induced by a gate voltage: Electrostatic approach. Phys. Rev. B 77, 155436 (2008).

43. Taychatanapat, T., Watanabe, K., Taniguchi, T. \& Jarillo-Herrero, P. Electrically tunable transverse magnetic focusing in graphene. Nat. Phys. 9, 225-229 (2013).

44. Ponomarenko, L. A. et al. Chaotic Dirac billiard in graphene quantum dots. Science 320, 356-358 (2008).

45. Han, M. Y., Brant, J. C. \& Kim, P. Electron transport in disordered graphene nanoribbons. Phys. Rev. Lett. 104, 056801 (2010).

46. Han, M. Y., Özyilmaz, B., Zhang, Y. \& Kim, P. Energy band-gap engineering of graphene nanoribbons. Phys. Rev. Lett. 98, 206805 (2007). 
47. Han, M. Y. \& Kim, P. Graphene nanoribbon devices at high bias. Nano Converg. 1, 1 (2014).

48. Tse, W.-K., Hwang, E. \& Das Sarma, S. Ballistic hot electron transport in graphene. Appl. Phys. Lett. 93, 023128 (2008).

49. Vaziri, S. et al. A graphene-based hot electron transistor. Nano Lett. 13, 1435-1439 (2013).

50. Zhang, P. \& Hung, D. M. An analytical model for ballistic diode based on asymmetric geometry. Appl. Phys. Res. 115, 204908 (2014).

51. Custer, J. P. Jr. et al. Ratcheting quasi-ballistic electrons in silicon geometric diodes at room temperature. Science 368, 177-180 (2020).

52. Stearns, J. \& Moddel, G. Nanoengineering: Fabrication, Properties, Optics, Thin Films, and Devices XVI. 11089, 1108903 (2019).

53. Uwanno, T. et al. Fully dry PMMA transfer of graphene on h-BN using a heating/ cooling system. 2d Mater. 2, 041002 (2015).

54. Pizzocchero, F. et al. The hot pick-up technique for batch assembly of van der Waals heterostructures. Nat. Commun. 7, 1-10 (2016).

55. Zomer, P. et al. Fast pick up technique for high quality heterostructures of bilayer graphene and hexagonal boron nitride. Appl. Phys. Lett. 105, 013101 (2014).

56. Wang, $\mathrm{H}$. et al. Hysteresis of electronic transport in graphene transistors. ACS Nano 4, 7221-7228 (2010).

57. Chiu, H.-Y., Perebeinos, V., Lin, Y.-M. \& Avouris, P. Controllable pn junction formation in monolayer graphene using electrostatic substrate engineering. Nano Lett. 10, 4634-4639 (2010).

58. Murali, R. et al. Breakdown current density of graphene nanoribbons. Appl. Phys. Lett. 94, 243114 (2009).

59. Han, M. Y. \& Kim, P. Graphene nanoribbon devices at high bias. Nano Converg. 1, 1-11 (2014).

\section{ACKNOWLEDGEMENTS}

This research was supported by a National Research Foundation of Korea (NRF) grant funded by the Korea government (MSIT) (NRF-2020R1A6A1A03043435 and 2020R1A2C1099862). This research was also supported by the Next-generation Engineering Researchers Development Program through NRF (NRF2019H1D8A2106002), and KETEP and MOTIE of the Republic of Korea (20194010000130). The author thanks Professor Zhigang Jiang, School of Physics, Georgia Institute of Technology, for experimental advice.

\section{AUTHOR CONTRIBUTIONS}

Research design, V.H.N. and Y.S.; Experiments and data analysis, D.C.N., M.K., M.H. V.H.N., Y.L., D.K., S.K., N.N., M.A.R., T.P.A.B., JJ., and Y.S.; Paper writing, V.H.N., S.K., and Y.S.

\section{COMPETING INTERESTS}

The authors declare no competing interests.

\section{ADDITIONAL INFORMATION}

Supplementary information The online version contains supplementary material available at https://doi.org/10.1038/s41699-021-00269-2.

Correspondence and requests for materials should be addressed to Yongho Seo.

Reprints and permission information is available at http://www.nature.com/ reprints

Publisher's note Springer Nature remains neutral with regard to jurisdictional claims in published maps and institutional affiliations.

(c) (i)

Open Access This article is licensed under a Creative Commons Attribution 4.0 International License, which permits use, sharing, adaptation, distribution and reproduction in any medium or format, as long as you give appropriate credit to the original author(s) and the source, provide a link to the Creative Commons license, and indicate if changes were made. The images or other third party material in this article are included in the article's Creative Commons license, unless indicated otherwise in a credit line to the material. If material is not included in the article's Creative Commons license and your intended use is not permitted by statutory regulation or exceeds the permitted use, you will need to obtain permission directly from the copyright holder. To view a copy of this license, visit http://creativecommons. org/licenses/by/4.0/.

(c) The Author(s) 2021 